\title{
Caracteríscas dos neonatos com dificuldade de mamada em um Hospital Universitário: Um estudo epidemiológico
}

\author{
Characteristics of newborns with difficulty breastfeeding in a University Hospital: An \\ epidemiological study \\ Características de los recién nacidos con dificultad de lactancia en un Hospital Universitario: Un \\ estudio epidemiológico
}

Recebido: 08/02/2022 | Revisado: 16/02/2022 | Aceito: 23/02/2022 | Publicado: 04/03/2022

Fernanda da Silva Tori ORCID: https://orcid.org/0000-0002-5140-3892 Universidade Estadual do Oeste do Paraná, Brasil E-mail: ferdstori@gmail.com

José Mohamud Vilagra ORCID: https://orcid.org./ 0000-0002-0885-724X Universidade Estadual do Oeste do Paraná, Brasil E-mail: jmvilagra@hotmail.com

Mario José de Rezende ORCID: https://orcid.org./0000-0003-4745-4440 Universidade Estadual do Oeste do Paraná, Brasil E-mail: mrezende7@yahoo.com.br

Marcelo Taglietti

ORCID: https://orcid.org./0000-0003-3650-3905 Universidade Estadual do Oeste do Paraná, Brasil E-mail: marcelotaglietti@gmail.com

Rebeka Crislaynne Timóteo de Carvalho Silva Barros ORCID: https://orcid.org./0000-0002-7243-8103 Universidade Estadual do Oeste do Paraná, Brasil E-mail: rebekacarvalho23@gmail.com Josivaldo de Mendonça Camilo ORCID: https://orcid.org./0000-0003-4268-7923 Universidade Estadual do Oeste do Paraná, Brasil E-mail: josivaldomcamilo@gmail.com

Thaynara Larissa Cagnini ORCID: https://orcid.org./0000-0002-5945-0933 Universidade Estadual do Oeste do Paraná, Brasil E-mail: thaynara.cagnini@gmail.com

\begin{abstract}
Resumo
Objetivo: Identificar as características epidemiológicas como idade gestacional, tipo de parto e o sexo dos neonato com dificuldade de mamada em um hospital escola no oeste do paraná. Metodologia: Estudo epidemiológico descritivo de fonte primária, desenvolvido na unidade materno infantil do Hospital Universitário do Oeste do Paraná (HUOP) no período entre março e setembro de 2021. Foram selecionado para o estudo neonatos com até 24horas de vida que apresentassem dificuldade de mamada relatada pela mãe e com score maior que sete na escala LATCH. Resultados: A amostra do estudo foi composta por setenta neonatos para o estudo, trinta e sete do sexo masculino nascidos de parto vaginal (52,9\%), trinta e três do sexo feminino nascidos de parto cesárea $(47,1 \%)$. A idade gestacional variou entre trinta e sete e quarenta e uma semanas de gestação, sendo a mais predominante trinta e nove semanas $(38,6 \%)$, seguido de trinta e oito $(24,3 \%)$ e quarenta semanas $(17,1 \%)$. Conclusão: O presente estudo demonstrou que as características dos neonatos com dificuldade de mamada de um hospital escola no oeste do paraná que apresenta-se na maioria por neonatos do sexo masculino, nascidos pela via de parto vaginal e com idade gestacional de 39 semanas.
\end{abstract}

Palavras-chave: Características de estudo epidemiológicos; Sucção; Aleitamento materno; Neonatos.

\footnotetext{
Abstract

Objective: To identify epidemiological characteristics such as gestational age, type of delivery and the sex of neonates with breastfeeding difficulties in a teaching hospital in western Paraná. Methodology: Descriptive epidemiological study from a primary source, carried out at the maternal and child care unit of the Hospital Universitário do Oeste do Paraná (HUOP) between March and September 2021. Neonates up to 24 hours old who had reported breastfeeding
} 
difficulties were selected for the study. by the mother and with a score greater than seven on the LATCH scale. Results: The study sample consisted of seventy newborns for the study, thirty-seven males born by vaginal delivery $(52.9 \%)$, thirty-three females born by cesarean section $(47.1 \%)$. Gestational age ranged between thirty-seven and forty-one weeks of gestation, with the most predominant being thirty-nine weeks (38.6\%), followed by thirty-eight $(24.3 \%)$ and forty weeks $(17.1 \%)$. Conclusion: The present study demonstrated that the characteristics of neonates with breastfeeding difficulties at a teaching hospital in western Paraná, which are mostly male neonates, born by vaginal delivery and with a gestational age of 39 weeks.

Keywords: Epidemiologic Study Characteristics; Suction; Breast feeding; Newborn.

\section{Resumen}

Objetivo: Identificar características epidemiológicas como la edad gestacional, el tipo de parto y el sexo de recién nacidos con dificultades para amamantar en un hospital escuela del oeste de Paraná. Metodología: Estudio epidemiológico descriptivo de fuente primaria, realizado en la unidad de atención materno-infantil del Hospital Universitário do Oeste do Paraná (HUOP) entre marzo y septiembre de 2021. del estudio por la madre y con una puntuación superior a siete en la escala LATCH. Resultados: La muestra de estudio estuvo constituida por setenta recién nacidos para el estudio, treinta y siete varones nacidos por parto vaginal $(52,9 \%)$, treinta y tres mujeres nacidas por cesárea $(47,1 \%)$. La edad gestacional osciló entre las treinta y siete y cuarenta y una semanas de gestación, siendo la más predominante la treinta y nueve semanas $(38,6 \%)$, seguida de la treinta y ocho $(24,3 \%)$ y cuarenta semanas $(17,1 \%)$. Conclusión: El presente estudio demuestra las características de los bebés nacidos con dificultad para la lactancia materna en un hospital escuela del oeste de Paraná, que es mayoritariamente atendido por bebés del sexo masculino, nacidos por parto vaginal y con edad gestacional de 39 semanas.

Palabras clave: Características de Estudios Epidemiológicos; Succión; Lactancia materna; Animales recién nacidos.

\section{Introdução}

O pré natal é um período importante para a intervenção de profissionais para contribuir positivamente na amamentação. A realização do pré natal iniciado no primeiro trimestre gestacional é muito importante pois a mãe recebe orientaçãoes gerais além de orientações relacionadas ao aleitamento materno favorecendo a amentação no período pós parto. (Oliveira, Lira, Batista, \& Lima, 2013)

O periodo neonatal começa nas primeiras quatro semanas de vida ( 0 á 28 dias incompletos) nesta idade o bebê requer cuidados adequados, uma maior vigilância e acompanhamento por parte do profissional de saúde a fim de garantir um melhor crescimento e desenvolvimento do neonato (Pinheiro et al., 2016).

A amamentação nas primeiras horas de vida é recomendada pela Organização Mundial da Saúde (OMS) sendo o leite materno o unico alimento necessário para os neonatos após o nascimento e deve ser oferecido exclusivamente, sem necessidade de outro complemento alimentar até os 6 meses de vida (Mosele et al., 2014).

A técnica de amamentação envolve o posicionamento correto do bebê na mama para estimular o reflexo de busca do bebê e é fundamental para uma amamentação bem sucedida. É necesário que o neonato abra bem a boca e empurre a língua para frente para pegar a mama e, em seguida, com a língua sob a aréola faça a sucção até extrair o leite da mama por meio de sucções lentas e profundas (Righard, 1998; Righard, \& Alade, 1992).

A dificuldade da mamada pode ser interferida por diversos fatores como fatores intrínsecos do bebê, como a pega e a deglutição e o peso ao nascimento, e fatores relacionados à mãe como falta de experiencia prévia com o aleitamento (primíparas), o tipo de mamilo, a via de parto, e a dor associada ao ato de amamentar (Ramalho, Martins, Lima, Andrade \& Koifman, 2019; Schafer et al., 2017; Silva et al., 2019; Urbanetto et al., 2018).

A forma correta da pega no ceio da mãe durante a amamentação é um importante fator para ganho de peso adequado e para que não ocorram lesões nas mamas (Ministério da saúde, 2015; Escobar et al., 2002; Faleiros, Trezza, \& Carandina, 2006). A técnica de amamentação ineficaz, que dificulta a sucção e o esvaziamento da mama, pode causar prejuízos na dinâmica da síntese de leite e problemas como ingurgitamento e mastite, os quais figuram entre os principais fatores associados à interrupção do AME (aleitamento materno exclusivo) (Barbosa et al., 2017). 
Dentre as ferramentas existentes na literatura que possibilitam auxiliar a avaliação do desempenho do bebê durante a mamada, destaca-se o LATCH Scoring System, bastante pesquisado, provavelmente pela sua praticidade (Altuntas et al., 2014; Sartorio et al., 2017). Cada letra do acrônimo LATCH representa uma característica: L (Latch) refere-se à qualidade da pega da criança na mama; A (Audible swallowing) refere-se à possibilidade de se ouvir a deglutição do bebê enquanto está mamando; $\mathrm{T}$ (Type of nipple) avalia o tipo de mamilo; $\mathrm{C}$ (Comfort) refere-se ao nível de conforto da mãe em relação à mama $\mathrm{e}$ ao mamilo; e H (Hold) refere-se ao fato de a mãe precisar ou não de ajuda para posicionar a criança. Cada um dos cinco componentes de avaliação do aleitamento materno recebe um escore numérico de 0 a 2, representando a mesma forma do Boletim de Apgar para uma pontuação máxima de 10 pontos (Jensen, Wallace, \& Kelsay, 1994).

O objetivo dessa pesquisa foi identificar as características epidemiológicas como idade gestacional, tipo de parto e o sexo dos neonato com dificuldade de mamada em um hospital escola no oeste do paraná.

\section{Metodologia}

A presente pesquisa trata-se de um estudo epidemiológico descritivo de fonte primária (Vieira \& Hossne, 2021) desenvolvido na unidade materno infantil do Hospital Universitário do Oeste do Paraná (HUOP) no período entre março e setembro de 2021. O estudo foi aprovado pelo Comitê de ética em Pesquisa da Universidade Estadual do Oeste do paraná pelo parecer número CAAE: 26035119.3.0000.0107. O questionário LATCH foi aplicado por um fisioterapeuta nos neonatos em internamento hospitalar na unidade materno infantil do HUOP que tivesssem de $12 \mathrm{~h}$ a 24 horas de vida e apresentassem dificuldade de mamada relatada pela mãe.

Critério de inclusão: neonatos de 12 á 24h de vida, dificuldade de mamada relatada pela mãe e com score LATCH maior que sete. Critérios de exclusão: neonatos nascidos com peso $\leq 1500 \mathrm{~g}$ (no nascimento); com idade gestacional $\leq 28$ semanas; má formação congênita; com histórico de cirurgias ou que apresentassem comprometimento neurológico, cardiaco ou alterações craniofaciais. Por questões de comunicação e também por questões de ordem legal não foi incluido no estudo indígenas, haitianos e estrangeiros em transito. Foram exluidos neonatos em isolamento de contato ou apresentassem febre, estivessem nessecitando de fototerapia ou em uso de oxigênio suplementar, além daqueles onde os responsáveis que não aceitassem assinar o TCLE.

Para a análise estatística os dados numéricos foram testados de acordo com a distribuição de normalidade e foram apresentados em média e desvio padrão. Já para os qualitativos foram realizadas distribuições de frequências e apresentados em frequência e porcentagem. Para comparação entre as frequências foi em empregado o teste de qui-quadrado. Os dados foram analisados no programa Social Package for the Social Sciences (SPSS), versão 24.0 e o nível de significância empregado foi o de $5 \%(\mathrm{p} \leq 0,05)$.

\section{Resultados e Discussão}

O presente estudo trata-se de um estudo epidemiológico descritivo de fonte primária, desenvolvido na maternidade do do HUOP no período entre julho de 2020 á fevereiro de 2022. Nesta pesquisa, participaram do estudo setenta recém nascidos a termo que apresentaram score do LACHT maior que 7, tendo em vista que foram selecionado bebês de 12 até 24h após o nascimento afim de obter uma amostra homogenea, todos os bebes apresentaram a mesma idade de vida ( 1 dia).

Os resultados representados na tabela 1 caracterizam a sexualidade dos neonatos. $\mathrm{O}$ sexo que mais predominou neste estudo foi o sexo masculino, com 37 participantes, caracterizando 52,9\% ja o sexo feminino incluiu 33 neonatos representando $47,1 \%$ (Tabela 1).

O gênero dos bebes com dificuldade de mamada desse estudo apresentou - se maior para o sexo masculino, assim como no estudo de Demezuk (2020) que avaliou o perfil dos recém nascidos com ictéricia neonatal, a ictericia pode estar 
relacionada com a dificuldade da ingesta do leite materno devido dificuldade de mamamada.

Já estudo realizado em 2020 no HUOP que avaliou nas suturas cranianas em 67 neonatos com dificuldade de amamentação apresentou 41,8\% do sexo masculino e 58,2\% do sexo feminino (Brasil \& Vilagra, 2021). Outro estudo em 2017 teve a presença maior de bebês do sexo feminino, nesta pesquisa foi analisado a eficacia do tratamento osteopático no aleitamento.

Tabela 1. Dados referente ao genero dos neonatos.

\begin{tabular}{lll}
\hline Sexo dos neonatos & Frequência & Porcentagem \\
\hline Masculino & 37 & $52,9 \%$ \\
Feminino & 33 & $47,1 \%$ \\
\hline
\end{tabular}

Fonte: Própria Autoria (2022).

A idade gestacional variou entre trinta e sete e quarenta e uma semanas de gestação, sendo a mais predominante trinta e nove semanas $(38,6 \%)$, seguido de trinta e oito $(24,3 \%)$ e quarenta semanas $(17,1 \%)$. Tabela 2.

O estudo de Brasil e Vilagra (2021) demonstrou que os bebês com dificuldade de mamada apresentaram uma idade gestacional de 38,2 $\pm 4,9$ semanas assim como a amostra do estudo de Demezuk (2020), realizado em um hospital universitário em 2020 que demonstrou que a maioria dos bebês eram a termo.

Existem estudos que apontam para a relação da dificuldade de amamentação de neonatos a termo porém é necessário mais estudos realizando a comparação entre neonatos a termo, prematuros e pós termo para verificar se há um nivel significancia entre essas variáveis.

Tabela 2. Dados referente a idade gestacional em semanas dos neonatos.

\begin{tabular}{lll}
\hline Idade Gestacional (semanas) & Frequência & Porcentagem \\
\hline $37 \mathrm{sem}$ & 8 & $11,4 \%$ \\
$38 \mathrm{sem}$ & 17 & $24,3 \%$ \\
$39 \mathrm{sem}$ & 30 & $42,9 \%$ \\
$40 \mathrm{sem}$ & 12 & $17,1 \%$ \\
$41 \mathrm{sem}$ & 3 & $4,3 \%$ \\
\hline
\end{tabular}

Fonte: Própria Autoria (2022).

O parto cesárea é considerado como fator de risco para o desmame precoce e necessita ser levado em consideração nas políticas de estímulo ao aleitamento materno (Oliveira, Lira, Batista, \& Lima, 2013). No estudo realizado, trinta e sete dos neonatos nasceram de parto vaginal, o que caracteriza 52,9\% e trinta e três nasceram de cesárea $(47,1 \%)$. (Tabela 3$)$

O tipo de parto não apresentou significância neste estudo, porém essa variante pode interferir na lactação principalmente quando falamos de parto cesárea, tendo em vista o desconforto físico da mãe, o uso de anestésicos e analgesicos que podem prolongar o tempo de hospitalização.

O estudo de Brasil e Vilagra (2021) mostrou uma maior frequência do tipo de parto vaginal quando comparado com cesária, o que vai ao encontro com o estudo realizado que paresentou uma maior frequencia também em partos vaginais.

A cesariana altera as respostas endócrinas na mãe e no bebê no período imediato após o parto, além de provocar dor na mãe e maior sonolência, condições que retardam e impõem dificuldades nas primeiras mamadas (Ford \& Labbok, 1990; Perez-Escamilla, Maulen-Radovan, Dewey, 1996) e as dificuldades com o início da amamentação pode gerar um desfecho 
negativo.

Portanto, a literatura apresenta o parto cesárea como fator de risco para o desmame precoce porém, conforme os dados obtidos neste estudo, não é possivel afirmar que o tipo de parto seja um fator determinante para a dificuldade na amamentação.

Tabela 2. Dados referente a via de parto dos neonatos.

\begin{tabular}{lll}
\hline Tipo de Parto & Frequência & Porcentagem \\
\hline Vaginal & 37 & $52,9 \%$ \\
Cesária & 33 & $47,1 \%$ \\
\hline
\end{tabular}

Fonte: Própria Autoria (2022).

\section{Conclusão}

O presente estudo caracterizou os neonatos com dificuldade de mamada de um hospital escola no oeste do paraná que apresenta-se na maioria por neonatos do sexo masculino (52,9\%), nascidos pela via de parto vaginal (52,9\%) e com idade gestacional de 39 semanas (42,9\%). Por se tratar de um hospital escola, os dados da atual pesquisa podem auxiliar na avaliação e tratamento dos neonatos com dificuldade de mamada visto que a dificuldade na amamentação é um fator importante de ser identificado nos primeiros dias pós-parto, para que não ocorra a interrupção precoce do aleitamento materno exclusivo.

Sugere-se que em estudos futuros sejam avaliados a inflência do tipo de parto em neonatos que apresentam dificuldade de mamada, pois este estudo foi de encontro com os demais apresentados na literatura e determinando esse fator, irá auxiliar os profissionais a delimitarem ainda mais as características desta população, podendo contribuir para o tratamento dos mesmo evitando o desmame precoce.

\section{Referências}

Altuntas, N., Turkyilmaz, C., Yildiz, H., Kulali, F., Hirfanoglu, I., Onal, E., ... Atalay, Y. (2014). Validity and reliability of the infant breastfeeding assessment tool, the mother baby assessment tool, and the LATCH scoring system. Breastfeeding Medicine, 9(4), 191-195.

Barbosa, G. E. F., Silva, V. B. da, Pereira, J. M., Soares, M. S., Medeiros Filho, R. dos A., Pereira, L. B., ... Caldeira, A. P. (2017). Dificuldades iniciais com a técnica da amamentação e fatores associados a problemas com a mama em puérperas. Revista Paulista de Pediatria, 35(3), 265-272.

Brasil, A. P. S., \& Vilagra, J. M. (2021). Morfologia das suturas cranianas em neonatos com dificuldade de amamentação. FAG Journal Of Health, 3(2), 119123.

Mosele, P. G., Santos, J. F., Godói, V. C., Costa, F. M., Toni, P. M., \& Fujinaga, C. I (2014). Instrumento de avaliação da sucção do recém-nascido com vistas a alimentação ao seio materno. Revista Centro de Especializaçao Em Fonoaudiologia Clinica, 16(5), $1548-1557$.

Demezuk, K.S.(2020). Perfil dos recém-nascidos internados com diagnóstico de icterícia neonatal e uma unidade de cuidados intermediários de um hospital universitário (Trabalho de conclusão de curso). Universidade Estadual do Oeste do Paraná, Cascavel, Paraná, Brasil.

Escobar, A. M. U., Ogawa, A. R., Hiratsuka, M., Kawashita, M. Y., Teruya, P. Y., Grisi, S., \& Tomikawa, S. O. (2002). Aleitamento materno e condições socioeconômico-culturais: fatores que levam ao desmame precoce. Revista Brasileira de Saúde Materno Infantil, 2(3), $253-261$.

Faleiros, F. T. V., Trezza, E. M. C., \& Carandina, L. (2006). Aleitamento materno: fatores de influência na sua decisão e duração. Revista de Nutrição, 19(5), 623-630.

Ford, K., \& Labbok, M. (1990). Who is breast-feeding? Implications of associated social and biomedical variables for research on the consequences of method of infant feeding. The American Journal of Clinical Nutrition, 52(3), 451-456.

Jensen, D., Wallace, S., \& Kelsay, P. (1994). LATCH:A Breastfeeding charting system and documentation Tool. Journal of Obstetric, Gynecologic \& Neonatal Nursing, 23(1), 27-32.

Ministério da Saúde. (2015). Saúde da criança: Aleitamento materno e alimentação complementar. 2.

Oliveira, M. G. O. A., Lira, P. I. C., Batista, M. F., \& Lima, M. C. (2013). Fatores associados ao aleitamento materno em dois municípios com baixo índice de desenvolvimento humano no Nordeste do Brasil. Revista Brasileira de Epidemiologia, 16(1), 178-189.

Perez-Escamilla, R., Maulen-Radovan, I., \& Dewey, K. G. (1996). The association between cesarean delivery and breast-feeding outcomes among mexican women. Americam Jorunal of Public Health, 86(6), 832-836. 
Research, Society and Development, v. 11, n. 3, e48911326754, 2022

(CC BY 4.0) | ISSN 2525-3409 | DOI: http://dx.doi.org/10.33448/rsd-v11i3.26754

Pinheiro, J. M. F., Tinoco, L. S., Rocha, A. S. S., Rodrigues, M. P., Lyra, C. O., \& Ferreira, M. Â. F. (2016). Atenção à criança no período neonatal: avaliação do pacto de redução da mortalidade neonatal no Rio Grande do Norte, Brasil. Ciência \& Saúde Coletiva, 21(1), 243-252.

Ramalho, A. A., Martins, F. A., Lima, T. A. S., Andrade, A. M., \& Koifman, R. J. (2019). Fatores associados à amamentação na primeira hor de vida em Rio Branco, Acre. DEMETRA: Alimentação, Nutrição \& Saúde, 14, e43809.

Righard, L. (1998). Are breastfeeding problems related to incorrect breastfeeding technique and the use of pacifiers and Bottles? Birth, $25(1), 40-44$.

Righard, L., \& Alade, M. O. (1992). Sucking technique and its effect on success of breastfeeding. Birth, 19(4), 185-189.

Sartorio, B. T., Coca, K. P., Marcacine, K. O., Abuchaim, É. S. V., \& Abrão, A. C. F. de V. (2017). Instrumentos de avaliação do aleitamento materno e seu uso na prática clínica. Revista Gaúcha de Enfermagem, 38(1), e64675.

Schafer, E. J., Campo, S., Colaizy, T. T., Mulder, P. J., Breheny, P., \& Ashida, S. (2017). First-time mothers' breast-feeding maintenance: role of experiences and changes in maternal perceptions. Public Health Nutrition, 20(17), 3099-3108.

Michelin, N. S., Nunes, H. R. C., Carvalhaes, M. A. B. L., \& Parada, C. M. G. L. (2021). The influence of gestational age at term on breastfeeding: a cohort study. Revista Da Escola de Enfermagem Da USP, 55, e20200381.

Silva, V. A. A. L., Caminha, M. F. C., Silva, S. L., Serva, V. M. S. B. D., Azevedo, P. T. A. C. C., \& Batista, M. F. (2019). Maternal breastfeeding: indicators and factors associated with exclusive breastfeeding in a subnormal urban cluster assisted by the Family Health Strategy. Jornal de Pediatria, 95(3), 298-305.

Urbanetto, P. D. G., Costa, A. R., Gomes, G. C., Nobre, C. M. G., Xavier, D. M., \& Jung, B. C. de. (2018). Facilidades e dificuldades encontradas pelas puérperas para amamentar. Revista de Pesquisa Cuidado é Fundamental Online, 10(2), 399-405.

Vieira, S., Hossne, W. S. (2021). Metodologia científica para a área da saúde (3a ed.). Rio de Janeiro, RJ: Guanabara Koogan. 